\title{
Cortical Current Density vs. Surface EEG for Event-Related Potential-based Brain-Computer Interface
}

\author{
Mohit Kumar Goel, Ricardo Chavarriaga, José del R. Millán
}

\begin{abstract}
In this paper we use the cortical current density based inverse solution to classify Event Related Potentials, in particular for error-related potentials elicited during a BrainComputer Interface experiment. We selected discriminant cortical sources for comparing classification performance with respect to surface EEG. We found that the data from estimated cortical sources achieves higher classification accuracy for most of the subjects. In addition, the inverse method exhibits consistently discriminant activity for the sources located over the anterior cingulate cortex region for different time points. This level of neurophysiological interpretation in terms of localisation of selected cortical sources is enabled with the use of inverse solution.
\end{abstract}

\section{INTRODUCTION}

Distributed inverse solutions allow to estimate intracranial source activity from the recorded surface Electroencephalograph (EEG) potentials [1]. Studies using this cortical activity for motor-imagery based Brain-Computer Interface (BCI) systems have shown that higher classification accuracies can be achieved in comparison to surface EEG data [2], [3]. This motivates us to study distributed inverse solution on different BCI experiments based on Event Related Potentials (ERPs).

In this paper we select error-related potential (ErrP) based ERP data [4], [5] to study the classification performance between surface EEG and cortical activity estimated using cortical current density (CCD) based distributed inverse solution [6]. A preliminary study on the feasibility of using CCD inverse solution for ERPs is reported in [7]. In our study, we found that the data from cortical sources not only increases classification accuracy for some subjects, but it also provides us an opportunity for neurophysiological interpretation of the ERP data in terms of active cortical sources. The inverse method provided localization information of the selected sources which is difficult to interpret from the surface EEG data directly.

\section{Materials AND Methods}

\section{A. CCD distributed inverse solution}

CCD distributed inverse solution is a linear method to estimate cortical activity from the measured EEG activity on the scalp [6]. The model assumes cortical activity in form of dipoles which are perpendicularly arranged on the cortical surface with fixed orientation. The geometry of the cortical mantle is obtained through an average head model from the

This work is supported by Swiss National Science Foundation, Project 200021-120293. Authors are affiliated to Defitech Chair in NonInvasive Brain-Machine Interface, Center for Neuroprosthetics, School of Engineering, École Polytechnique Fédérale de Lausanne (EPFL), Switzerland, CH-1015. e-mail: mohitkumar.goeldepfl.ch
MNI institute [8]. The cortical mantle is divided into 3013 vertices, each representing a dipolar source. Mathematically, the CCD activity ( $\mathbf{x})$ is estimated using a linear transformation of surface EEG potential (b) at a given time $t$ as

$$
\mathbf{x}(t)=\mathbf{G b}(t)
$$

where $\mathbf{G}$ is a matrix that represents the inverse of the forward model emulating the electrical propagation properties from the cortical sources to surface EEG potentials. For more details on the CCD model and inverse solutions, please refer [1], [6], [9]. The linear projection from EEG potential to $\mathrm{CCD}$ vortices tends to un-mix the EEG signals, which results in an improved spatial resolution and signal-to-noise ratio. Hence classifiers computed using this activity may ameliorate recognition performances of brain activity.

\section{B. ErrP-based ERP experiment}

We use the data from an experiment aimed to study error potentials during brain-computer interaction, where subjects were asked to monitor movement of a cursor moving towards a target on the screen [5]. The cursor movement is termed erroneous whenever it moves away from the target. The objective of this experiment is to correctly identify the types of cursor movement from the surface EEG signals into two classes: correct and erroneous. Erroneous movements were randomly generated with probability $P_{E r r}=0.2$ in order to elicit error-related EEG activity. In each recording session, subjects monitored 10 runs of 15 targets (approx. 90 single trials per block). Two recording sessions per subject were performed on different days with a difference ranging from 2 months to 2 years. Fig. 1 shows the error-minus-correct grand average (FCz electrode), which is characterized by two fronto-central positive peaks appearing at $200 \mathrm{~ms}$ and 320 $\mathrm{ms}$ as well as fronto-central negativity near $250 \mathrm{~ms}$ after receiving error feedback from the agent.

\section{Preprocessing}

The ErrP recording was performed using Biosemi ActiveTwo system with a sampling frequency of $512 \mathrm{~Hz}$. Sixty-four electrodes were used according to the standard 10/20 international system. The common average reference (CAR) was used as spatial filter followed by a $1-10 \mathrm{~Hz}$ band-pass filter and then the epochs corresponding to erroneous and correct cursor movements were extracted. Downsampled data (factor of 16) between the time window $200 \mathrm{~ms}$ and $450 \mathrm{~ms}$ after the stimulus presentation was considered for further analysis because ErrP was most prominent in this time window as shown in Fig. 1. 


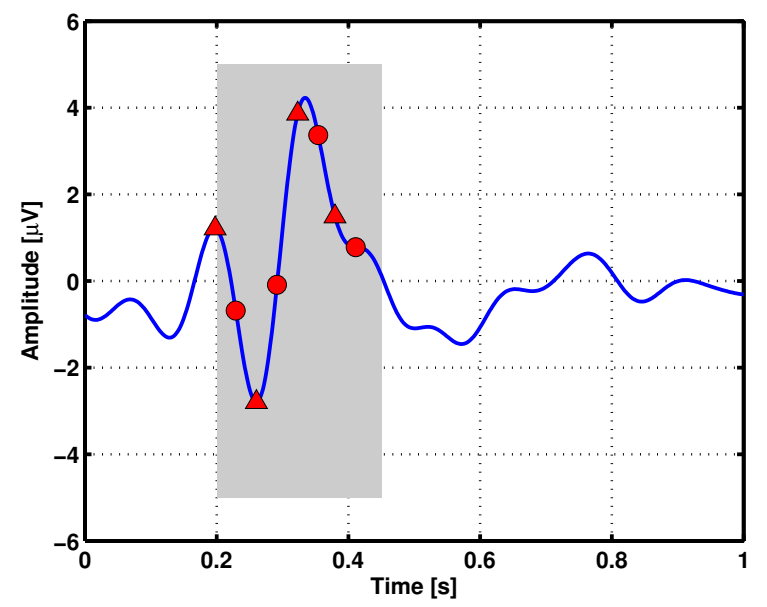

Fig. 1. Grand average ERP, error-minus-correct at channel $\mathrm{FCz}$ for six subjects. Time window $\mathrm{T}=\left[\begin{array}{ll}200 & 450\end{array}\right] \mathrm{ms}$ is highlighted in gray with points in red used for classification purpose. Triangular points are used for topographic analysis as discussed in section III-B.

\section{Classification}

For classifying the spatio-temporal features of the ErrP data, we make single sub-decisions for the spatial features at every time point (i.e. we train a separate classifier per time point) and finally combine all of them to make a single decision for the whole trial. This procedure enabled us to interpret our results with cortical sources as discussed in section III-B.

For the surface EEG data, we use a Fisher linear discriminator (FLD) [10] to estimate a spatial weighting vector $\mathbf{w}\left(t_{i}\right)$, $i=[1,2, \ldots, T]$ represents time points shown in Fig. 1 that maximally discriminate the trials for two conditions, correct versus erroneous. The FLD projected value is represented as $y(t)$. Sub-decision for each time point $t$ is obtained as a probability $P\left(\omega_{j} \mid y(t)\right)$ for the class $\omega_{j},(j=1,2)$. The probability $P\left(\omega_{j} \mid y(t)\right)$ is computed as the likelihood,

$$
P\left(\omega_{j} \mid y(t)\right)=\frac{1}{\sqrt{2 \pi \sigma_{j}(t)}} e^{-\frac{\left(y(t)-\mu_{j}(t)\right)^{2}}{2 \sigma_{j}^{2}(t)}}
$$

where $\mu_{j}(t)$ and $\sigma_{j}(t)$ are mean and covariance for the data of class $\omega_{j}$ in the FLD canonical space. The final decision for a trial is made on the basis of all the probabilities over the selected time window,

$$
P\left(\omega_{j} \mid \mathbf{y}\right)=\frac{1}{T} \sum_{i=1}^{T} P\left(\omega_{j} \mid y\left(t_{i}\right)\right)
$$

Classification using CCD requires to deal with a feature space with size corresponding to 3013 cortical values. This number is significantly high to use FLD as proper estimation of the covariance would require a prohibitively large number of training trials. To solve this problem, we perform a feature selection procedure based on the discriminative power of each source and then we take a decision by combining classification ability of a subset of these sources with highest discriminant power. We use a separability score ( $r^{2}$ measure) to quantify the discriminability of each source and select top $D$ highest ranking cortical sources. The value of $\mathrm{D}$ is chosen based on a statistical test as discussed in section III. For classification purposes, we assign a probability $P\left(\omega_{j} \mid x_{i}(t)\right)$ to each selected cortical source $i(=1,2, \ldots, D)$ at time $t$ using the same likelihood formula as in equation 2 . The probability $P\left(\omega_{j} \mid \mathbf{x}(t)\right)$ at time $t$ is computed by combining output for all the sources,

$$
P\left(\omega_{j} \mid \mathbf{x}(t)\right)=\frac{1}{D} \sum_{i=1}^{D} P\left(\omega_{j} \mid x_{i}(t)\right)
$$

Final decision for a trial follows the same combination rule as described in equation 3 .

It is important to note that each cortical source activity by itself is a linear weighted combination of surface EEG potentials. Thus in principle, every cortical source carries information from all the EEG electrodes. If we perform an equivalent spatial procedure of source selection with surface EEG potentials, we will end up in selecting a subset of EEG potential values, which we want to avoid as we are interested is using information from all the EEG electrodes and not just restricting to some electrodes.

\section{RESULTS}

\section{A. Classification Accuracy}

The data recorded for session 1 was used for training the classifiers and session 2 for testing them. With this approach, we want to show the applicability of our classification system on two different days of recording. This factor is important for BCI-systems as the trained classifier is expected to function with high accuracy on different days of recording [11].

For the purpose of classification using cortical sources, we wanted to choose sufficient number of sources to make a robust decision system. We performed Kruskal-Wallis statistical test on the probabilities obtained for the test trials (for each class separately) with $D$ ranging from 10 to 150 and found no significant differences in the range [50 150]. For this preliminary study we choose $D=100$.

Table I presents the classification results for 6 subjects. We separately compute the classification accuracy for the two classes. We also present the value of area under the ROC curve for each method which provided a single value performance measure for the maximum likelihood based classification for unbalanced classes presented in this paper. The results clearly show that for all subjects, the CCD method achieves higher accuracy in classifying trials belonging to correct class which is statistically significant (Kruskal-Wallis, $p<0.05)$ except subject 4 . When the accuracy of error class is concerned, the CCD method significantly increase accuracy for subject 1 (Kruskal-Wallis, $p<0.05$ ) and achieve similar classification performance with the surface EEG for subjects 3,5 and 6 . We only found 2 subjects (subjects 2 and 4) where the surface EEG-based system gives significantly higher accuracy when recognizing the erroneous trials (Kruskal-Wallis, $p<0.05$ ), although one of them performs around random level (subject 4). The above higher 
TABLE I

Percentage ACCURACY OF CLASSIFICATION AND AREA UNDER ROC CURVE WITH TWO METHODS.

\begin{tabular}{|c|c|c|c|c|c|c|}
\hline \multirow{2}{*}{ Subj } & \multicolumn{3}{|c|}{ CCD method } & \multicolumn{3}{c|}{ Surface EEG } \\
\cline { 2 - 7 } & Corr(\%) & Err(\%) & AUC & Corr(\%) & Err(\%) & AUC \\
\hline 1 & 90.1 & 73.5 & 0.901 & 85.6 & 61.5 & 0.791 \\
2 & 96.2 & 71.3 & 0.950 & 66.7 & 83.7 & 0.847 \\
3 & 87.4 & 78.7 & 0.905 & 85.0 & 79.8 & 0.896 \\
4 & 80.4 & 36.1 & 0.670 & 72.3 & 50.9 & 0.709 \\
5 & 65.8 & 77.2 & 0.843 & 49.9 & 75.0 & 0.723 \\
6 & 90.1 & 65.6 & 0.896 & 89.4 & 59.8 & 0.859 \\
\hline
\end{tabular}

classification results obtained for CCD method over surface EEG is also support by the respective higher AUC values.

\section{B. Topographic analysis of cortical sources}

Based on the accuracy of CCD method, we found that subject 1 has the best performance. On the contrary, subject 4 has the worst performance as the detection of erroneous trials has fallen to $36.11 \%$. We consider only these two subjects for our further analysis due to space limitations.

In Fig. 2 we show the localization of selected cortical sources at time points highlighted by triangular marks in Fig. 1. Information from these sources is used to compute the combined probability in equation 4.

For subject 1 , at time $t=200 \mathrm{~ms}$ after stimulus presentation, we find the selected sources are localized over the region of cingulate cortex with some activation originating from the anterior cingulate cortex (ACC) region as well. According to FMRI studies, ACC region is consistently involved in error-related cognitive process [12]. We can see that the distribution of selected cortical sources change over time. For the time $t=343 \mathrm{~ms}$, we find a high concentration of selected sources over the cingulate cortex which reduces to a more frontal region at time $t=414 \mathrm{~ms}$. This shows the change in activity level of cortical region over time for error awareness.

However, for subject 4, we find a more scattered distribution of selected sources over different time instances. Discriminant sources are selected over cingulate cortex for the first time at $t=343 \mathrm{~ms}$. Although this distribution is not as dense as subject 1, it still shows that error awareness is undertaken by sources over cingulate cortex at that time instance. For the time points $t=343,414 \mathrm{~ms}$ some of the discriminant sources are selected from the prefrontal cortex region. This anomaly could be possible due to ocular artifacts.

In addition to these maps, a combined cortical map in Fig. 3 shows the frequency of occurrence of the selected cortical sources. These maps highlight the region in the brain which contributes to the maximum information in discriminating the two classes across the whole time window. The map for subject 1 shows that the region above ACC is a significant contributor in decision making process. However, for subject 4 we find two different regions in decision making, one over cingulate cortex and another over prefrontal cortex. In addition to subjects 1 and 4 , we found

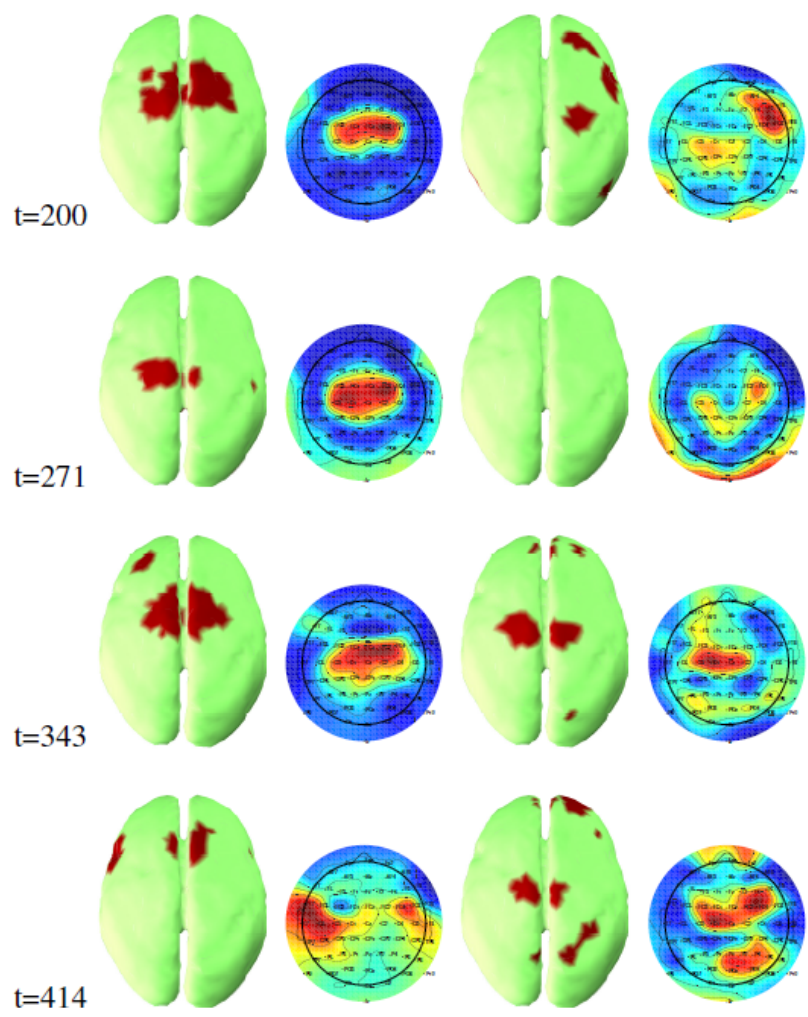

Subject 1

Fig. 2. Selected sources on CCD maps $(D=100)$ vs relative weights of electrodes on surface EEG topographic maps for subject 1 and 4 Highlighted time points (ms) in Fig. 1 are considered for this analysis. The nose is facing upwards for all the maps.

the distribution of selected sources was well localized over cingulate cortex for subjects 2 and 3. In subjects 5 and 6 we found scattered distribution with localisation over cingulate cortex and parietal cortex.

\section{Topographic analysis of FLD weights}

The FLD weights mentioned in section II-D define a canonical projection that maximally separates two classes at a selected time point. These weights determine the importance of each EEG electrode at a given time point for classification of data. We plot the absolute value of these weights in form of surface EEG topographic maps along with the maps of cortical region for the same subjects (see Fig. 2). The color scheme for the maps is normalized such that red represents maximum absolute weight and blue represents minimum absolute weight.

For subject 1, we find a strong focus for the electrodes over cingulate region of the brain for $t=200,271,343 \mathrm{~ms}$. The relative importance of EEG electrodes in this region seems to be similar till $t=414 \mathrm{~ms}$ where we find that there are two foci over left and right temporal regions. With respect to localization information in cortical maps, the weight distribution over EEG electrodes limits our ability 


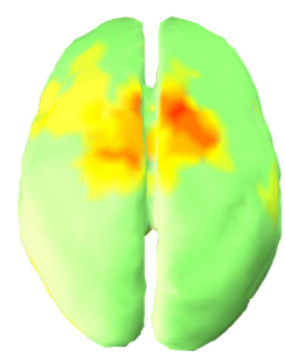

Subject 1

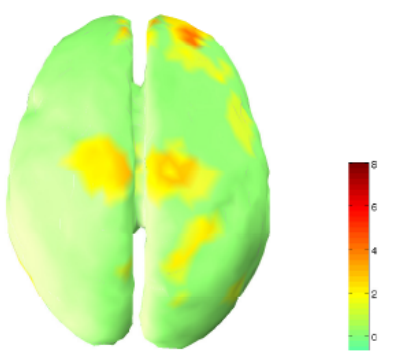

Subject 4
Fig. 3. Combined map showing the frequency of selection of the cortical sources across the selected time window. Nose is facing upwards.

to interpret classification procedure for time instances $t=$ 200, 271, $343 \mathrm{~ms}$.

For subject 4 , we see that a clear focus over cingulate region at time $t=200,271 \mathrm{~ms}$. The weighting of EEG electrodes keep changing over time which shows the activation of different regions of the brain. This high spatial variability with time could be the result of unsustained activation pattern across different trials which leads to a low classification accuracy for this subject in comparison to subject 1 .

\section{CONClusion And Future Work}

In this paper we performed single-trial classification on ERP-based BCI using data from the estimated cortical sources and compared it with surface EEG. For each time point we computed a probability of occurrence of a class and combined these probabilities over time to make a single decision. We found that the data from the estimated cortical sources achieves higher classification accuracy for most of the subjects. The premise of performance increase with the use of inverse solution over surface EEG for MI-based BCI experiments seems to be true with this ERP-based BCI experiment as well. This suggest that we need to test this inverse method for other ERP experiments to validate the claim.

The inverse solution for cortical source activity estimation is developed for neurophysiological studies in source localization. Its application for BCI purpose is relatively new. In this paper, we used CCD inverse solution for better classification performance and also for interpreting the localization of selected cortical sources using cortical maps. This localization aspect of inverse solution provides us with more neurophysiological assistance in interpreting the results. We found that for the subject 1 who has high accuracy of recognition in ErrP experiment also has more number of cortical sources selected around cingulate cortex in comparison to the subject 4 which has lower performance. This distribution of cortical sources is more stable across the relevant time window for subject 1 than for subject 4 . Thus, lower contribution of sources from the cingulate area to the recorded scalp EEG may be related to classification accuracy for subject 4 .

We found a variability in localization of selected sources over time for the same task. We speculate that some of these selected sources are not involved in error cognition and might be responsible for other neurophysiological task. A possible approach would be to perform classification only based on cortical sources known to be involved in the process of interest (i.e. ACC for error-related processes). Alternatively, a combined approach can be devised where both the discriminant power and the neurophysiological relevance are taken into account for the feature selection. In this regard we propose to incorporate our neurophysiological knowledge in selecting these sources instead of a fixed number which might result in higher accuracy of recognition. This procedure may be related to beamforming approaches where neurophysiologically relevant sources are used to design spatial filters for EEG data [13]. We leave this analysis for our future studies.

\section{ACKnOWLEDGMEnTS}

Authors thank IRCCS Fondazione Santa Lucia, Rome, for the CCD model. Thanks also to G. Garipelli for his valuable suggestions and discussion.

\section{REFERENCES}

[1] R. Grech, T. Cassar, J. Muscat, K. P. Camilleri, S. G. Fabri, M. Zervakis, P. Xanthopoulos, V. Sakkalis, and B. Vanrumste, "Review on solving the inverse problem in EEG source analysis," J Neuro Eng and Rehab, vol. 5, p. 25, 2008.

[2] R. Grave de Peralta Menendez, S. L. Gonzalez Andino, L. Perez, P. Ferrez, and J. del R. Millán, "Non-invasive estimation of local field potentials for neuroprosthesis control," Cogn Process, vol. 6, pp. 5964, 2005.

[3] M. Congedo, F. Lotte, and A. Lécuyer, "Classification of movement intention by spatially filtered electromagnetic inverse solutions," Phy in Med Bio, vol. 51, no. 8, pp. 1971-1989, 2006.

[4] P. W. Ferrez and J. del R. Millán, "Error-related EEG potentials generated during simulated brain-computer interaction," IEEE Tran Biomed Eng, vol. 55, pp. 923-929, 2008.

[5] R. Chavarriaga and J. del R. Millán, "Learning from EEG errorrelated potentials in noninvasive brain-computer interfaces." IEEE Trans Neural Syst Rehabil Eng, vol. 18, no. 4, pp. 381-388, Aug 2010.

[6] F. Cincotti, D. Mattia, F. Aloise, S. Bufalari, L. Astolfi, F. D. V. Fallani, A. Tocci, L. Bianchi, M. G. Marciani, S. Gao, J. del R. Millan, and F. Babiloni, "High-resolution EEG techniques for brain-computer interface applications," J Neurosci Methods, vol. 167, no. 1, pp. 31-42, Jan 2008.

[7] G. Garipelli, R. Chavarriaga, F. Cincotti, F. Babiloni, and J. del R. Millán, "Discriminative channel selection method for the recognition of anticipation related potentials from CCD estimated cortical activity," in Proc of IEEE Int Ws on MLSP, 2009.

[8] M. Fuchs, J. Kastner, M. Wagner, S. Hawes, and J. S. Ebersole, "A standardized boundary element method volume conductor model," Clin Neurophysiol, vol. 113, no. 5, pp. 702-712, 2002.

[9] F. Babiloni, C. Babiloni, L. Locche, F. Cincotti, P. M. Rossini, and F. Carducci, "High-resolution electro-encephalogram: Source estimates of Laplacian-transformed somatosensory-evoked potentials using a realistic subject head model constructed from magnetic resonance images," Med Biol Eng Comput, vol. 38, no. 5, pp. 512-519, Sep 2000.

[10] R. O. Duda, P. E. Hart, and D. G. Stork, Pattern Classification (2nd Edition), 2nd ed. Wiley-Interscience, November 2000.

[11] G. Dornhege, J. del R. Millán, T. Hinterberger, D. J. McFarland, and K. R. Müller, Toward Brain-Computer Interfacing (Neural Information Processing). The MIT Press, 2007.

[12] C. B. Holroyd and M. G. H. Coles, "The neural basis of human error processing: Reinforcement learning, dopamine, and the error-related negativity." Psychol Rev, vol. 109, no. 4, pp. 679-709, Oct 2002.

[13] M. Grosse-Wentrup, C. Liefhold, K. Gramann, and M. Buss, "Beamforming in noninvasive brain-computer interfaces." IEEE Trans Biomed Eng, vol. 56, no. 4, pp. 1209-1219, Apr 2009. 